\title{
MiRNA Expression Profile in the Airways Is Altered during Pulmonary Exacerbation in Children with Cystic Fibrosis-A Preliminary Report
}

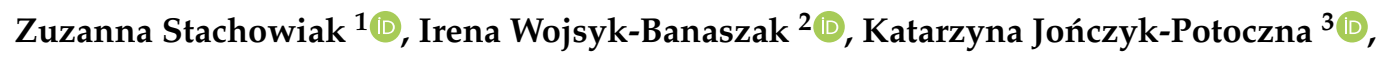 \\ Beata Narożna ${ }^{1}$, Wojciech Langwiński ${ }^{1}$, Zdzisława Kycler ${ }^{2}$, Paulina Sobkowiak ${ }^{2}$, \\ Anna Bręborowicz ${ }^{2}$ and Aleksandra Szczepankiewicz ${ }^{1, *}$ \\ 1 Molecular and Cell Biology Unit, Department of Paediatric Pulmonology, Allergy and Clinical Immunology, \\ Poznan University of Medical Sciences, 60-572 Poznań, Poland; zuzastachowiak9@gmail.com (Z.S.); \\ b.narozna@gmail.com (B.N.); wlangwinski654@gmail.com (W.L.) \\ 2 Department of Paediatric Pulmonology, Allergy and Clinical Immunology, Poznan University of Medical \\ Sciences, 60-572 Poznań, Poland; iwojsyk@ump.edu.pl (I.W.-B.); kyclerzdzislawa@interia.pl (Z.K.); \\ paulina-25@tlen.pl (P.S.); abreborowicz@wp.pl (A.B.) \\ 3 Department of Paediatric Radiology, Poznan University of Medical Sciences, 60-572 Poznań, Poland; \\ potocznak@op.pl \\ * Correspondence: alszczep@gmail.com; Tel.: +48-618-547-643
}

Received: 26 May 2020; Accepted: 15 June 2020; Published: 16 June 2020

\begin{abstract}
MicroRNAs are small non-coding RNAs that regulate immune response and inflammation. We assumed that miRNAs may be involved in the immune response during cystic fibrosis pulmonary exacerbations (CFPE) and that altered expression profile in the airways and blood may underlie clinical outcomes in CF pediatric patients. Methods: We included 30 pediatric patients diagnosed with cystic fibrosis. The biologic material (blood, sputum, exhaled breath condensate) was collected during pulmonary exacerbation and in stable condition. The miRNA expression profile from blood and sputum $(n=6)$ was done using the next-generation sequencing. For validation, selected four miRNAs were analyzed by qPCR in exosomes from sputum supernatant and exhaled breath condensate $(n=24)$. NGS analysis was done in Base Space, correlations of gene expression with clinical data were done in Statistica. Results: The miRNA profiling showed that four miRNAs (miR-223, miR-451a, miR-27b-3p, miR-486-5p) were significantly altered during pulmonary exacerbation in CF patients in sputum but did not differ significantly in blood. MiRNA differently expressed in exhaled breath condensate (EBC) and sputum showed correlation with clinical parameters in CFPE. Conclusion: MiRNA expression profile changes in the airways during pulmonary exacerbation in CF pediatric patients. We suggest that miRNA alterations during CFPE are restricted to the airways and strongly correlate with clinical outcome.
\end{abstract}

Keywords: miRNA expression; exhaled breath condensate; sputum; severity; pulmonary exacerbation

\section{Introduction}

Cystic fibrosis (CF) is a common autosomal recessive disorder caused by mutations in the cystic fibrosis transmembrane conductance regulator (CFTR) gene. It is a multiorgan disease that affects the pancreas, gastrointestinal and reproductive tracts and lungs [1]. Common pulmonary symptoms include deposition of thick mucus that leads to severe airflow obstruction, chronic inflammation and chronic airway infection [2]. Lung disease is characterized by intermittent episodes of acute worsening of respiratory symptoms, pulmonary exacerbations (CFPE) [3] that cause lung function decline and contribute to disease progression, poor quality of life and shortened life expectancy in CF patients. 
Retention of thick mucus in the airways facilitates frequent pulmonary infections (caused by such pathogens as P. aeruginosa, S. aureus, $H$. influenzae) [4]. However, pulmonary exacerbations do not usually result from increased bacterial density within the airways $[5,6]$, and more severe symptoms are caused by chronic enhanced airway inflammation that may even precede infection. Chronic inflammation in the airways leads to airway epithelium damage and activates repair processes contributing to remodeling and subepithelial fibrosis that further impair lung function. Current CFPEs are the leading cause of morbidity and mortality $[7,8]$, indicating the need for biomarker discovery.

Published data looking for CFPE biomarkers either in blood or the airways suggest many immunologically active molecules, such as TNF $\alpha$, IL-8, myeloperoxidase (MPO), calprotectin, C reactive protein $(\mathrm{CRP})$, correlated with clinical deterioration $[9,10]$. However, none of them were specific for CF pulmonary exacerbation. Moreover, peripheral biomarkers may not truly reflect the local inflammatory response in the lungs [11]. Available data suggest that sputum is a reliable source of inflammatory biomarkers, including circulating miRNA [12,13]. Less is known about the markers in exhaled breath condensate (EBC) [14].

MicroRNA are small (21-23 bp), non-coding RNAs that modulate the expression of various proteins via post-transcriptional inhibition of gene expression. They are well-known regulators of immune responses and chronic inflammation in respiratory diseases, including asthma, COPD or idiopathic pulmonary fibrosis (as reviewed by $[15,16]$ ). Moreover, they are expressed not only inside the cells but are also stably present in extracellular space i.e., body fluids such as plasma, sputum, bronchoalveolar lavage fluid (BALF) and EBC. Previous studies of miRNA in the course of CF reported a few miRNAs associated with CFTR activity (either decreased expression e.g., miR-509-3p, miR-145, miR-223 or increased expression, e.g., miR-138) [17] as well as a pulmonary exacerbation. Krause et al. studied the latter and found that the expression of the Mirc1/Mir17-92 cluster correlated with pulmonary exacerbation, but only in sputum samples and not in plasma [18]. Altered miRNA expression also affects innate immune responses in the CF lung. Previous reports showed that mir-126 was significantly downregulated in CF bronchial brushings and thus influenced the expression of Target of Myb1 (TOM1) membrane trafficking protein $[19,20]$. However, previous studies on the role of miRNA in the pediatric CF population are limited. The recent analysis focused on the miRNA expression profile and gender differences in CF children [21], but the studies investigating miRNA expression profile in CF pulmonary exacerbation are lacking.

Taking into account that miRNAs are regulators of immune inflammatory responses and that enhanced chronic inflammation plays a significant role in pulmonary exacerbations in cystic fibrosis, we aimed to investigate if miRNA expression differs between exacerbation and stable period of disease and if altered expression profile in the airways may underlie clinical outcome in CF pediatric patients.

\section{Materials and Methods}

\subsection{Study Design}

The prospective cohort of 30 pediatric patients, girls and boys aged 6-18 years diagnosed with cystic fibrosis and admitted to the Department of Pulmonology, Pediatric Allergy and Immunology, was enrolled in the project. Patients were either experiencing a pulmonary exacerbation or in a stable stage of disease (control visit). The Bioethics Committee approved the study (no. 386/17). All pediatric participants and their parents gave written consent.

\subsection{Clinical Analysis}

CF was diagnosed based on typical clinical presentation, two positive sweat chloride tests and the presence of two pathogenic mutations of CFTR. Pulmonary exacerbation was defined according to the EuroCareCF working group [22,23]. In all patients, we assessed: blood morphology, inflammatory proteins (CRP), microbiologic sputum culture, chest imaging (Brasfield score), spirometry and disease severity (with Shwachman-Kulczycki score). The data were collected, as described previously [24]. 
Sputum samples were collected during spontaneous expectoration. Samples were prepared by the addition of Sputolysin (Merck, Kenilworth, NJ, USA) and centrifuged to separate the cell pellet from the supernatant. For further analysis, sputum supernatant was used. We used the Turbo DECCS device (Medivac) to collect EBC according to ERS recommendation [25]. From all patients, the same amount ( $3 \mathrm{~mL}$ ) of EBC was collected. Whole blood samples were drawn to tubes with EDTA anticoagulant, and after the immediate addition of Lysis Buffer (Macherey-Nagel, Düren, Germany), samples were frozen at $-80^{\circ} \mathrm{C}$ for further analysis.

\subsection{Molecular Analysis}

MiRNA samples from blood and sputum were extracted using NucleoSpin RNA blood kit (Macherey-Nagel). Exosomes from sputum supernatant and exhaled breath samples were precipitated using a miRCURY exosome cell/urine/CSF Isolation Kit (Qiagen, Hilden, Germany). MicroRNA was extracted using RNeasy mini kit (Qiagen) and RNeasy MinElute spin columns according to protocol to obtain miRNA fraction. The quantity of miRNA in the samples was measured using microRNA assay kit (Thermo Fisher Scientific, Waltham, MA, USA). The miRNA expression profile from the whole blood and sputum samples of 6 CF patients during exacerbation and stable period was performed using next-generation sequencing. Libraries were generated from $50 \mathrm{ng}$ of miRNA sample using TruSeq small RNA library preparation kit (Illumina, San Diego, CA, USA) following the manufacturer's instructions. Libraries were validated and quantified using high sensitivity DNA screen tape (Agilent, Santa Clara, CA, USA) on Tape Station 2200 (Agilent) and run on MiniSeq sequencer (Illumina) with 50-nt single-end reads. Differential miRNA expression analysis was done in Base Space software. The miRNA genes that showed the most significant differences between CF exacerbation and stable period in miRNA expression profiling from sputum were analyzed using qPCR in the validation $\mathrm{CF}$ cohort ( $n=24$ samples) in sputum supernatants and exhaled breath condensate. For reverse transcription, we used MystiCq microRNA cDNA Synthesis Mix (Sigma-Aldrich, St. Louis, MO, USA). Quantitative PCR was done using MystiCq microRNA SYBR Green qPCR ReadyMix (Sigma Aldrich) and microRNA assays for miR-486-5p, miR-223, miR-451a, miR-27b. Expression analysis was conducted on the 7900HT fast-real time PCR system (Applied Biosystems, Foster City, CA, USA). Differential expression results from qPCR were compared using Data Assist software (Thermo Fisher Scientific) with a relative quantification method based on the global normalization algorithm.

\subsection{Statistical Analysis}

The data distribution was analyzed using the Shapiro-Wilk test. Normally distributed data were analyzed using parametric tests and data that deviated from normal distribution were analyzed using nonparametric tests. The correlation of miRNA expression and interval variables (lung function parameters, Shwachman-Kulczycki score, Brasfield score, neutrophil counts, CRP level) compared between exacerbation and stable period was done using the Spearman correlation test. The comparison of miRNA expression and nominal variables (presence of bacterial or fungal infection, presence of exacerbation) was performed using analysis of variance (one-way or two-way ANOVA) in Statistica v.12 (Statsoft, Cracow, Poland).

\section{Results}

\subsection{A Clinical Description of the Analyzed Group}

Clinical characteristics of the patients based on biologic material (exhaled breath condensate, sputum supernatant) are given in Tables 1 and 2 . The exacerbation group was similar in age and gender to the patients during a stable period. Chronic Pseudomonas aeruginosa colonization was rarely observed in our population $(\leq 25 \%)$ and not significantly different between exacerbation vs. stable groups. The lung function between groups was not significantly different, but the groups differed in clinical outcomes such as disease severity (SK score, Brasfield score). The treatment was uniform for the CF 
patients, depending on clinical symptoms. Patients chronically infected with Pseudomonas aeruginosa were treated with inhaled antibiotics, mostly colistimethate sodium, and two patients received inhaled tobramycin. All the patients received dornase alfa, all patients with pancreatic insufficiency received pancreatic enzymes and none were treated with CFTR modulators.

Table 1. Characteristics of the Cystic fibrosis (CF) patients (exhaled breath condensate (EBC) samples).

\begin{tabular}{cccc}
\hline \multicolumn{4}{c}{ Mean $( \pm$ SD) or Number (\%) } \\
\hline Variable & Stable & Exacerbation & $p$ Value \\
\hline Number of subjects & 12 & 12 & - \\
Female & $6(50 \%)$ & $7(58 \%)$ & 1.000 \\
Age (years) & $12.4( \pm 3.5)$ & $13.0( \pm 3.4)$ & 0.706 \\
F508 del homozygous & $4(33 \%)$ & $6(50 \%)$ & 0.680 \\
F508del heterozygous & $5(42 \%)$ & $2(17 \%)$ & 0.371 \\
BMI $\left(\right.$ kg/m $\left.{ }^{2}\right)$ & $17.8( \pm 2.0)$ & $17.1( \pm 2.3)$ & 0.421 \\
Diabetes (including under observation) & $3(25 \%)$ & $5(42 \%)$ & 0.667 \\
Pancreatic insufficiency & $10(83 \%)$ & $11(92 \%)$ & 1.000 \\
P. aerugionsa colonization & $2(17 \%)$ & $4(33 \%)$ & 0.365 \\
P. aeruginosa current infection & $0(0 \%)$ & $3(25 \%)$ & 0.217 \\
S. aureus colonization & $9(75 \%)$ & $11(92 \%)$ & 0.590 \\
FEV $\%$ pred. & $98.3( \pm 19.8)$ & $82.8( \pm 14.9)$ & 0.041 \\
FVC $\%$ pred. & $84.8( \pm 12.5) *$ & 0.058 \\
Shwachman-Kulczycki score (median) & $90.0(65.0-95.0)$ & $85.0(40.0-85.0)$ & 0.041 \\
Brasfield score (median) & $23.0(11.0-25.0)$ & $13.0(5.0-22.0)$ & 0.027 \\
\hline
\end{tabular}

${ }^{*}$ spirometry baseline result. BMI-body mass index; FEV1-forced expiratory volume in the first second, FVC-forced vital capacity.

Table 2. Characteristics of the CF patients (sputum samples).

\begin{tabular}{cccc}
\hline & Mean $( \pm \mathbf{S D})$ or Number $(\%)$ & \\
\hline Variable & Stable & Exacerbation & $p$ Value \\
\hline Number of subjects & 11 & 13 & - \\
Female & $5(45 \%)$ & $6(43 \%)$ & 1.000 \\
Age (years) & $11.8( \pm 3.1)$ & $13.7( \pm 3.8)$ & 0.200 \\
F508 del homozygous & $5(45 \%)$ & $10(71 \%)$ & 0.241 \\
F508del heterozygous & $3(27 \%)$ & $3(21 \%)$ & 1.000 \\
BMI $\left(\right.$ kg/m $\left.{ }^{2}\right)$ & $18.5( \pm 3.1)$ & $18.7( \pm 2.4)$ & 0.885 \\
Diabetes (including under observation) & $4(36 \%)$ & $7(50 \%)$ & 0.689 \\
Pancreatic insufficiency & $9(81 \%)$ & $12(92 \%)$ & 0.615 \\
P. aeruginosa colonization & $2(18 \%)$ & $0(0 \%)$ & 0.113 \\
P. aeruginosa current infection & $2(18 \%)$ & $6(43 \%)$ & 0.234 \\
S. aureus colonization & $8(73 \%)$ & $13(93 \%)$ & 0.288 \\
FEV $\%$ pred. & $96.0( \pm 20.7)$ & $79.6( \pm 24.6)$ & 0.095 \\
FVC $\%$ pred. & & $84.2( \pm 22.2) *$ & 0.194 \\
Brasfield score & $94.8( \pm 15.1)$ & $83.2( \pm 19.4)$ & 0.121 \\
Shwachman-Kulczycki score (median) & $90.0(60.0-95.0)$ & $70.0(40.0-85.0)$ & 0.263 \\
& $20.2( \pm 3.3)$ & $15.4( \pm 3.9)$ & 0.009 \\
\hline
\end{tabular}

${ }^{*}$ spirometry baseline result. BMI-body mass index; FEV1-forced expiratory volume in the first second, FVC-forced vital capacity.

\subsection{Comparison of miRNA Expression Profile between Exacerbation and Stable Period}

Next-generation sequencing of the whole blood miRNA profile showed that out of 186 miRNAs expressed in blood, CF patients did not differ significantly between exacerbation and the stable period in (Figure 1a). Comparative analysis of the miRNA expression profile in sputum showed 
significant differences in the expression of 9 miRNAs (including mature miRNAs: miR-223-3p, miR-451a, miR-27b-3p and miR-486-5p and their isomiRs: 2 isomiRs of miR-486-5p, one of miR-451a and one of miR-223-3p.) between exacerbation and stable period out of 158 expressed in sputum (Figure 1b). Three of them, miR-27b, miR-223 and miR-19b, showed a decrease, whereas the other two (miR-486-5p, miR-451a) showed increased expression during exacerbation.
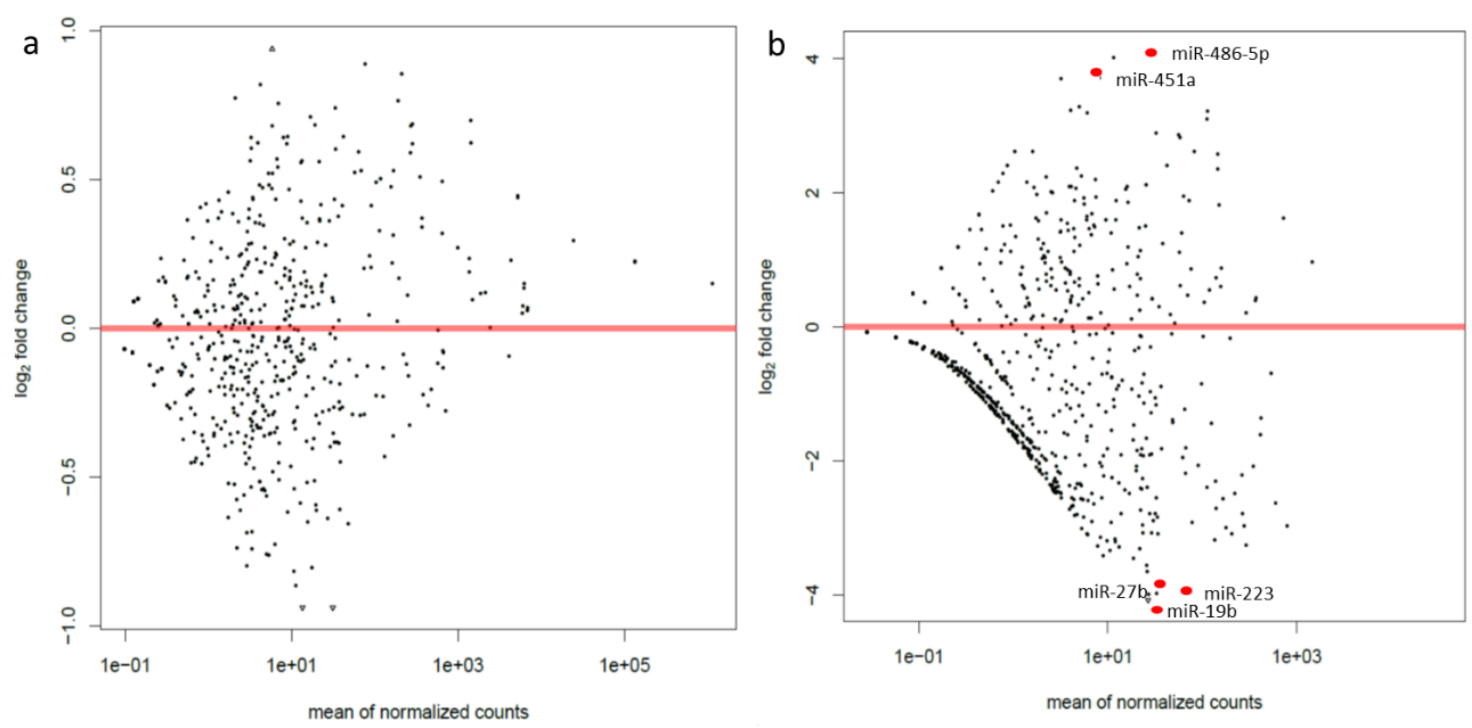

Figure 1. MA-plot between exacerbation and stable period of the gene expression counts (a) for the whole blood and (b) for sputum. Significantly altered miRNAs are marked as red dots (DeSeq).

Four mature miRNAs differentially expressed in sputum (miR-223-3p, miR-451a, miR-27b-3p and miR-486-5p) were selected for qPCR analysis in the sputum supernatant from 24 patients and exhaled breath condensate samples from 24 patients. One miRNA, miR-19b, showed very low expression in the sequencing experiment, so we excluded it from $\mathrm{qPCR}$ validation. During exacerbation, we observed the highest increase in expression for miR-486-5p in both EBC and sputum supernatant samples, but the difference compared to a stable period was not significant. For miR-223-3p, we found that decreased expression during exacerbation in the sequencing experiment was not confirmed in the validation cohort.

Expression of exosomal miR-223-3p was higher in CF patients during exacerbation in EBC and sputum samples, but the difference was not significant $(p>0.05)$. In EBC during exacerbation miR-451a was more abundant than in stable period, but the difference was not statistically significant $(p=0.41)$. In sputum supernatant, miR-451a was decreased during exacerbation than stable period, yet the difference did not reach significance. The only miRNA that showed decreased expression in both EBC and sputum samples during exacerbation was miR-27b, but this decrease was not significant. The results are shown in Table 3.

Table 3. MicroRNA (MiRNA) levels in patients during pulmonary exacerbation in reference to stable period.

\begin{tabular}{ccccc}
\hline miRNA & Expression (EBC) & $p$ Value & Expression (Sputum) & $p$ Value \\
\hline miR-223-3p & $\uparrow$ & 0.177 & $\uparrow$ & 0.411 \\
miR-451a & $\uparrow$ & 0.342 & $\downarrow$ & 0.406 \\
miR-27b-3p & $\downarrow$ & 0.160 & $\downarrow$ & 0.312 \\
miR-486-5p & $\uparrow$ & 0.656 & $\uparrow$ & 0.341 \\
\hline
\end{tabular}




\subsection{Correlation of miRNA Expression with Clinical Parameters}

\subsubsection{MiR-223-3p}

The higher expression of this miRNA in EBC correlated with Aspergillus infection in the airways during pulmonary exacerbation in CF patients $(p=0.02)$. Similar to EBC, we observed higher levels of this miRNA in sputum supernatants in children infected with Aspergillus ( $p=0.08$ ) (Figure 2), but not with symptoms severity (Shwachman-Kulczycki score, Brasfield score) or lung function.
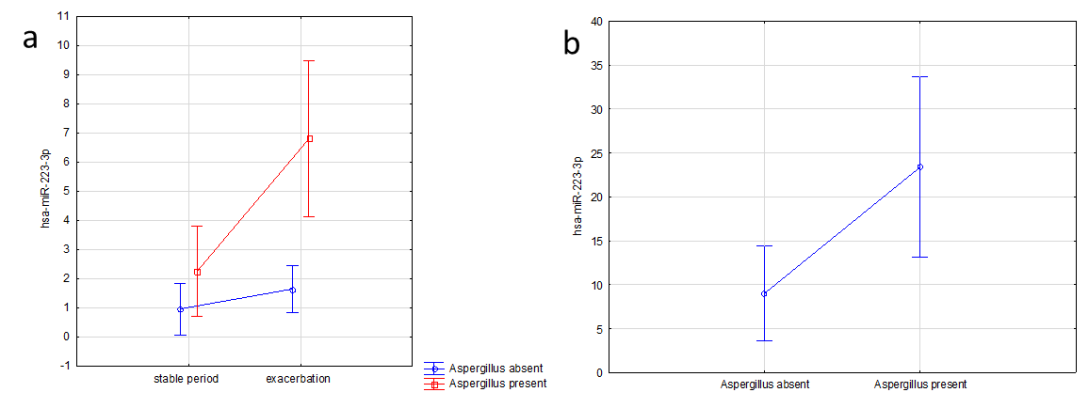

Figure 2. The association of miR-223-3p expression and clinical parameters in CF patients (a) in EBC in relation to Aspergillus infection and exacerbation ( $\mathrm{F}=5.953, p=0.024,2$-way analysis of variance); (b) in sputum supernatant in relation to Aspergillus infection ( $\mathrm{F}=6.714, p=0.017$, one-way analysis of variance).

\subsubsection{MiR-451a}

We observed that CF patients infected with $H$. influenzae had significantly higher miR-451a EBC expression during exacerbation $(p=0.02)$. In contrast, the level of expression in uninfected CF patients was similar and independent of exacerbation. We also observed a significant inverse correlation between miR-451a EBC expression and the Brasfield score $\left(r^{2}=-0.53, p=0.01\right)$ (Figure 3$)$, but the other clinical parameters did not correlate with the expression of this miRNA. We found no significant differences in miR-451a expression with clinical parameters in sputum.
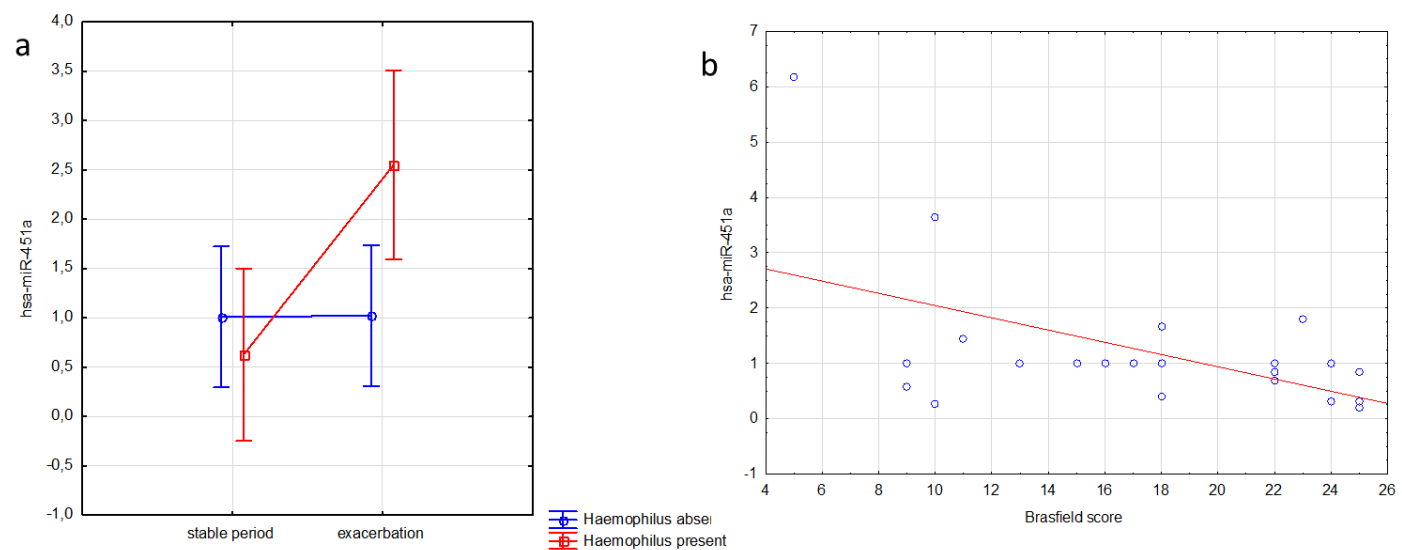

Figure 3. Association of miR-451a expression in EBC and clinical parameters in CF patients, (a) in relation to Hemophilus infection and exacerbation ( $\mathrm{F}=5.760 ; p=0.024$, 2-way analysis of variance);

(b) correlation with the Brasfield score ( $\mathrm{r}=-0.526 ; p=0.001$, Spearman correlation).

\subsubsection{MiR-27b-3p}

The expression of miR-27b in EBC positively correlated with lung function parameters: FEV1 $\left(r^{2}=0.669, p=0.077\right)$ and FVC $\left(r^{2}=0.637, p=0.006\right)$ during pulmonary exacerbation. 
Current infection with Pseudomonas aeruginosa was associated with decreased expression of miR-27b-3p in sputum supernatant during exacerbation than the patients in a stable period $(p<0.0001)$. It remained unaltered in patients without Pseudomonas infection (Figure 4).
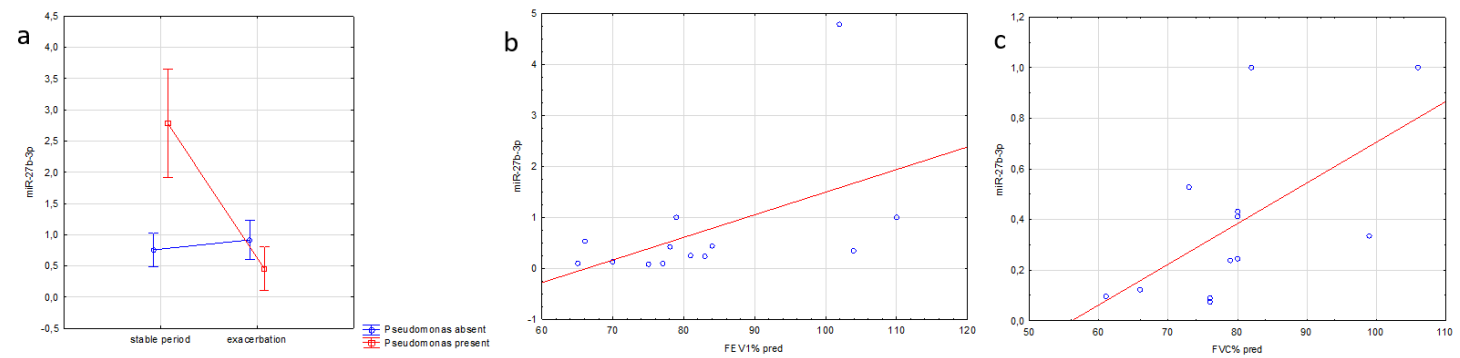

Figure 4. Association of miR-27b expression and clinical parameters in CF patients, (a) in sputum correlation with Pseudomonas infection during exacerbation $(\mathrm{F}=25.267, p<0.001$, 2-way analysis of variance); (b) in EBC correlation with FEV1 ( $\mathrm{r}=0.510 ; p=0.077)$ and $(\mathbf{c})$ FVC ( $\mathrm{r}=0611 ; p=0.034)$ during exacerbation (Spearman correlation).

\subsubsection{MiR-486-5p}

The expression of this miRNA in EBC did not correlate significantly with analyzed clinical parameters in CF patients. In sputum, miR-486-5p expression was higher in CF patients colonized with Pseudomonas aeruginosa $(p=0.044)$. Further analysis showed an inverse correlation between this miRNA and Shwachman-Kulczycki score $\left(\mathrm{r}^{2}=-0.419, p=0.047\right)$ as well as the Brasfield score $\left(\mathrm{r}^{2}=-0.459\right.$, $p=0.031)$ and lung function (FVC) $\left(\mathrm{r}^{2}=-0.421, p=0.046\right)$. On the other hand, results for two parameters of peripheral inflammation, CRP and neutrophilia, positively correlated with miR-486-5p expression in CF patients during PE for CRP $\left(\mathrm{r}^{2}=0.592, p=0.027\right)$ and neutrophilia $\left(\mathrm{r}^{2}=0.772\right.$, $p=0.001$ ) (Figure 5).
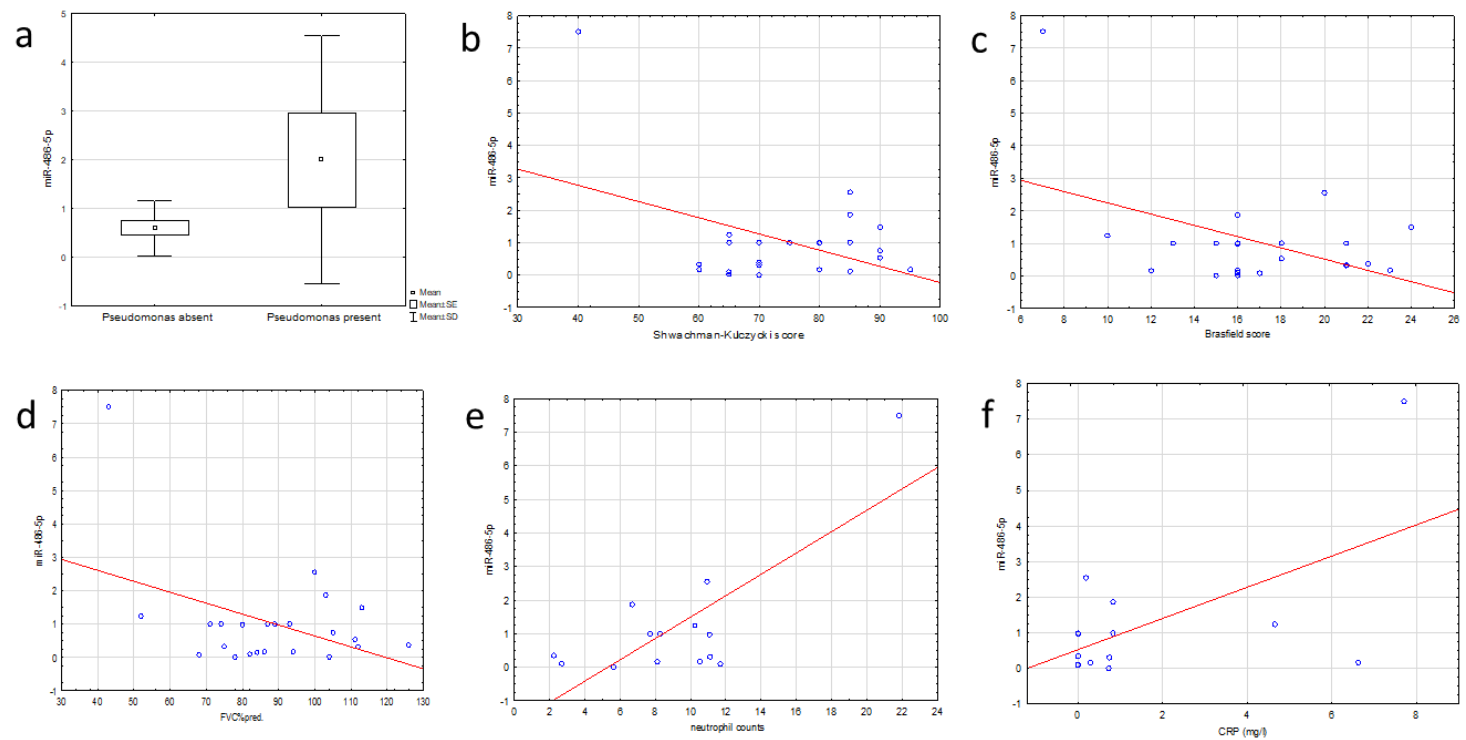

Figure 5. Association of miR-486-5p expression in sputum and clinical parameters in CF patients (a) in relation to Pseudomonas infection ( $p=0.044$, 1-way analysis of variance); (b) correlation with Shwachman-Kulczycki score $(\mathrm{r}=-0.42, p=0.048),(\mathrm{c})$ correlation with Brasfield score $(\mathrm{r}=-0.45$, $p=0.031)$; (d) Spearman correlation with FVC ( $\mathrm{r}=-0.451, p=0.045)$; correlation with peripheral markers of inflammation: (e) neutrophil counts $(\mathrm{r}=0.772 ; p=0.001)$ and (f) CRP levels $(\mathrm{r}=0.592$; $p=0.025)$ during exacerbation (Spearman correlation). 


\section{Discussion}

The main finding of this study is the altered miRNA expression profile in the airways, but not in blood, during pulmonary exacerbation. We also found that altered miRNAs (miR-223-3p, miR-451a, miR-27b, miR486-5p) correlate with clinical outcomes (bacterial infections, the severity of symptoms, lung function, peripheral inflammation) in sputum and exhaled breath condensate of CF patients.

Previous studies showed that miR-223 is a negative regulator of neutrophil activation and chemotaxis in experimental models of inflammatory diseases. This miRNA mediates intracellular neutrophil action as well as extracellular inflammation responses in the lungs [26,27]. In CF, neutrophilic inflammation in the lung correlated with more severe lung disease [28,29]. Previous data indicated that Aspergillus colonization may be associated with reduced lung function and that Aspergillus infection increased neutrophil count and elevated the levels of inflammatory cytokines (e.g., IL-8) in BALF [30,31]. In our study, we found increased expression of miR-223 during exacerbation with concurrent Aspergillus infection. Thus, miR-223-3p upregulation may be a response to infection and enhanced airway inflammation induced by Aspergillus.

Regarding miR-451a, a previous study showed increased expression of this miRNA in BALF from healthy subjects in the lower lung lobes compared to the upper lobes [32]. In silico analysis indicated several targets for miR-451a, e.g., macrophage migration inhibitory factor (MIF) and matrix metalloproteinase-2 (MMP-2), that are innate immunity mediators [33]. MIF is a proinflammatory molecule that enhances Gram-negative inflammatory responses, and its high level accelerates end-organ injury in CF [33]. In our study, we found the highest miR-451a expression in EBC during exacerbation in patients infected with Hemophilus influenzae. Infection with these Gram-negative bacteria in cystic fibrosis often co-occurs with PE [34]. An inverse correlation of miR-451a expression with the Brasfield score suggests that increased expression in the upper airways (EBC) may be a marker of disease progression assessed by radiological changes. Interestingly, the expression of miR-451a in CF exacerbation slightly decreased in sputum and did not correlate with Hemophilus infection. This observation indicates that miRNA alterations are site-specific within the respiratory tract and differ between body fluids from the upper and lower airways.

The expression of miR-27b-3p decreased during pulmonary exacerbation in both EBC and sputum supernatant, however, this change was not significant. Previous studies indicated this miRNA silences the immune response by reducing cytokine secretion, thus inhibiting local macrophage recruitment. Its expression increased during acute infection, e.g., sepsis preventing the spreading of inflammation [35]. This action was confirmed in an animal model of Mycobacterium tuberculosis infection [36]. Infected mice overexpressed in the airways miR-27b that suppressed the production of pro-inflammatory factors and excessive inflammation. Similarly, we observed that Pseudomonas aeruginosa infection correlated with overexpression of miR-27b in sputum in a stable period. Thus, its increase seems to suppress the exaggerated inflammatory response. Its significant decrease during exacerbation correlated with enhanced inflammation induced by Pseudomonas infection. Increased expression of miR-27b in EBC also associated with better lung function parameters, e.g., FEV 1 or FVC, suggesting that airway inflammation suppressed by miR-27b overexpression may translate into improved lung function in CF.

Previous data regarding mir-486-5p showed its increased plasma expression in CF patients than healthy controls [37]. We confirmed its expression in whole blood and also in sputum and EBC. A recent study showed that mice with induced acute lung injury presented higher miR-486-5p expression, whereas its inhibition with antagomir blocked inflammatory response and apoptosis, thus reducing lung injury [38]. Our results showed an increased expression of exosomal miR-486-5p in the sputum of CF patients infected with $P$. aeruginosa. Its expression inversely correlated with symptoms severity (Shwachman-Kulczycki and Brasfield scores) and lung function indicating a worse course of lung disease. Therefore, the increased local expression of this microRNA may be a marker of worse prognosis in CF patients. However, further studies are needed to confirm this assumption. 
One of the main limitations of the study is small sample size of our group and the fact that patients from the exacerbation group were different from patients from the stable group.

Another limitation may be high variability in clinical and miRNA expression data comparisons in the correlation analysis and to address this issue, we used nonparametric tests to minimize the influence of outliers on the results interpretation.

\section{Conclusions}

In conclusion, we found the altered miRNA expression profile in the airways of CF patients. These changes correlate with the clinical outcomes and suggest the involvement of miRNA regulation in pulmonary exacerbation. Our study also indicates the potential of non-invasively collected material from the airways (exhaled breath condensate) that may be a source of extracellular biomarkers of $\mathrm{CF}$ pulmonary exacerbation in children.

Author Contributions: Z.S. participated in the study design, sample collection, molecular analysis and data interpretation and drafting the manuscript; I.W.-B. participated in the study design, recruitment of patients, data analysis and the revision of the manuscript; K.J.-P. participated in the study design, clinical assessment of patients, data analysis and the revision of the manuscript; B.N. participated in sample collection, data analysis and preparation of the manuscript; W.L. participated in the data analysis and curation and preparing the manuscript; Z.K. participated in the clinical supervision and preparing the manuscript, P.S. participated in the clinical supervision and preparing the manuscript, A.B. participated in the study design, clinical supervision and preparing the manuscript; A.S. designed the study, performed molecular analysis, interpreted the data, revised and edited the manuscript, acquired the funding and administered the project. All authors have read and agreed to the published version of the manuscript.

Funding: This research was funded by the Polish National Science Center, grant no. 2016/22/E/NZ5/00383 (A. Szczepankiewicz).

Conflicts of Interest: The authors declare no conflict of interest. The funders had no role in the design of the study; in the collection, analyses or interpretation of data; in the writing of the manuscript or in the decision to publish the results.

\section{References}

1. Dos Santos, A.L.M.; de Melo Santos, H.; Nogueira, M.B.; Tavora, H.T.O.; de Lourdes Jaborandy Paim da Cunha, M.; de Melo Seixas, R.B.P.; de Freitas Velloso Monte, L.; de Carvalho, E. Cystic Fibrosis: Clinical Phenotypes in Children and Adolescents. Pediatric Gastroenterol. Hepatol. Nutr. 2018, 21, 306314. [CrossRef]

2. Belkin, R.A.; Henig, N.R.; Singer, L.G.; Chaparro, C.; Rubenstein, R.C.; Xie, S.X.; Yee, J.Y.; Kotloff, R.M.; Lipson, D.A.; Bunin, G.R. Risk factors for death of patients with cystic fibrosis awaiting lung transplantation. Am. J. Respir. Crit. Care Med. 2006, 173, 659-666. [CrossRef] [PubMed]

3. Waters, V.; Ratjen, F. Pulmonary Exacerbations in Children with Cystic Fibrosis. Ann. Am. Thoracic Soc. 2015, 12 (Suppl. S2), S200-S206. [CrossRef]

4. Zemanick, E.T.; Hoffman, L.R. Cystic Fibrosis: Microbiology and Host Response. Pediatric Clin. N. Am. 2016, 63, 617-636. [CrossRef] [PubMed]

5. Stressmann, F.A.; Rogers, G.B.; Marsh, P.; Lilley, A.K.; Daniels, T.W.; Carroll, M.P.; Hoffman, L.R.; Jones, G.; Allen, C.E.; Patel, N.; et al. Does bacterial density in cystic fibrosis sputum increase prior to pulmonary exacerbation? J. Cyst. Fibros. Off. J. Eur. Cyst. Fibrosis Soc. 2011, 10, 357-365. [CrossRef] [PubMed]

6. Zemanick, E.T.; Wagner, B.D.; Harris, J.K.; Wagener, J.S.; Accurso, F.J.; Sagel, S.D. Pulmonary exacerbations in cystic fibrosis with negative bacterial cultures. Pediatric Pulmonol. 2010, 45, 569-577. [CrossRef] [PubMed]

7. Bhatt, J.M. Treatment of pulmonary exacerbations in cystic fibrosis. Eur. Respir. Rev. Off. J. Eur. Respir. Soc. 2013, 22, 205-216. [CrossRef]

8. Jacquot, J.; Tabary, O.; Clement, A. Hyperinflammation in airways of cystic fibrosis patients: What's new? Ex. Rev. Mol. Diagn. 2008, 8, 359-363. [CrossRef]

9. Gray, R.D.; Imrie, M.; Boyd, A.C.; Porteous, D.; Innes, J.A.; Greening, A.P. Sputum and serum calprotectin are useful biomarkers during CF exacerbation. J. Cyst. Fibrosis Off. J. Eur. Cyst. Fibros. Soc. 2010, 9, 193-198. [CrossRef] [PubMed] 
10. Colombo, C.; Costantini, D.; Rocchi, A.; Cariani, L.; Garlaschi, M.L.; Tirelli, S.; Calori, G.; Copreni, E.; Conese, M. Cytokine levels in sputum of cystic fibrosis patients before and after antibiotic therapy. Pediatric Pulmonol. 2005, 40, 15-21. [CrossRef] [PubMed]

11. Gray, R.D.; Downey, D.; Taggart, C.C. Biomarkers to monitor exacerbations in cystic fibrosis. Ex. Rev. Respir. Med. 2017, 11, 255-257. [CrossRef] [PubMed]

12. Maes, T.; Cobos, F.A.; Schleich, F.; Sorbello, V.; Henket, M.; De Preter, K.; Bracke, K.R.; Conickx, G.; Mesnil, C.; Vandesompele, J.; et al. Asthma inflammatory phenotypes show differential microRNA expression in sputum. J. All. Clin. Immunol. 2016, 137, 1433-1446. [CrossRef] [PubMed]

13. Sagel, S.D.; Wagner, B.D.; Anthony, M.M.; Emmett, P.; Zemanick, E.T. Sputum biomarkers of inflammation and lung function decline in children with cystic fibrosis. Am. J. Respir. Crit. Care Med. 2012, 186, 857-865. [CrossRef]

14. Mendes, F.C.; Paciencia, I.; Ferreira, A.C.; Martins, C.; Rufo, J.C.; Silva, D.; Cunha, P.; Farraia, M.; Moreira, P.; Delgado, L.; et al. Development and validation of exhaled breath condensate microRNAs to identify and endotype asthma in children. PLoS ONE 2019, 14, e0224983. [CrossRef] [PubMed]

15. Stolzenburg, L.R.; Harris, A. The role of microRNAs in chronic respiratory disease: Recent insights. Biol. Chem. 2018, 399, 219-234. [CrossRef]

16. Gon, Y.; Shimizu, T.; Mizumura, K.; Maruoka, S.; Hikichi, M. Molecular techniques for respiratory diseases: MicroRNA and extracellular vesicles. Respirology 2020, 25, 149-160. [CrossRef]

17. Sonneville, F.; Ruffin, M.; Guillot, L.; Rousselet, N.; Le Rouzic, P.; Corvol, H.; Tabary, O. New insights about miRNAs in cystic fibrosis. Am. J. Pathol. 2015, 185, 897-908. [CrossRef]

18. Krause, K.; Kopp, B.T.; Tazi, M.F.; Caution, K.; Hamilton, K.; Badr, A.; Shrestha, C.; Tumin, D.; Hayes, D., Jr.; Robledo-Avila, F.; et al. The expression of Mirc1/Mir17-92 cluster in sputum samples correlates with pulmonary exacerbations in cystic fibrosis patients. J. Cyst. Fibros. Off. J. Eur. Cyst. Fibros. Soc. 2018, 17, 454-461. [CrossRef]

19. Oglesby, I.K.; Bray, I.M.; Chotirmall, S.H.; Stallings, R.L.; O’Neill, S.J.; McElvaney, N.G.; Greene, C.M. miR-126 is downregulated in cystic fibrosis airway epithelial cells and regulates TOM1 expression. J. Immunol. 2010, 184, 1702-1709. [CrossRef]

20. McKiernan, P.J.; Greene, C.M. MicroRNA Dysregulation in Cystic Fibrosis. Med. Inflamm. 2015, $2015,529642$. [CrossRef]

21. Mooney, C.; McKiernan, P.J.; Raoof, R.; Henshall, D.C.; Linnane, B.; McNally, P.; Glasgow, A.M.A.; Greene, C.M. Plasma microRNA levels in male and female children with cystic fibrosis. Sci. Rep. 2020, 10, 1141. [CrossRef] [PubMed]

22. Szentpetery, S.; Flume, P.A. Optimizing outcomes of pulmonary exacerbations in cystic fibrosis. Curr. Opin. Pulm. Med. 2018, 24, 606-611. [CrossRef] [PubMed]

23. Bilton, D.; Canny, G.; Conway, S.; Dumcius, S.; Hjelte, L.; Proesmans, M.; Tummler, B.; Vavrova, V.; De Boeck, K. Pulmonary exacerbation: Towards a definition for use in clinical trials. Report from the EuroCareCF Working Group on outcome parameters in clinical trials. J. Cyst. Fibros. Off. J. Eur. Cyst. Fibros. Soc. 2011, 10 (Suppl. S2), S79-S81. [CrossRef]

24. Wojsyk-Banaszak, I.; Sobkowiak, P.; Jonczyk-Potoczna, K.; Narozna, B.; Langwinski, W.; Szczepanik, M.; Kycler, Z.; Breborowicz, A.; Szczepankiewicz, A. Evaluation of Copeptin during Pulmonary Exacerbation in Cystic Fibrosis. Med. Inflamm. 2019, 2019, 1939740. [CrossRef]

25. Horvath, I.; Hunt, J.; Barnes, P.J.; Alving, K.; Antczak, A.; Baraldi, E.; Becher, G.; van Beurden, W.J.; Corradi, M.; Dekhuijzen, R.; et al. Exhaled breath condensate: Methodological recommendations and unresolved questions. Eur. Respir. J. 2005, 26, 523-548. [CrossRef] [PubMed]

26. Dorhoi, A.; Iannaccone, M.; Farinacci, M.; Fae, K.C.; Schreiber, J.; Moura-Alves, P.; Nouailles, G.; Mollenkopf, H.J.; Oberbeck-Muller, D.; Jorg, S.; et al. MicroRNA-223 controls susceptibility to tuberculosis by regulating lung neutrophil recruitment. J. Clin. Investig. 2013, 123, 4836-4848. [CrossRef] [PubMed]

27. Hall, C.H.T.; Campbell, E.L.; Colgan, S.P. Neutrophils as Components of Mucosal Homeostasis. Cell. Mol. Gastroenterol. Hepatol. 2017, 4, 329-337. [CrossRef] [PubMed]

28. Tirouvanziam, R. Neutrophilic inflammation as a major determinant in the progression of cystic fibrosis. Drug News Perspect. 2006, 19, 609-614. [CrossRef] 
29. Pillarisetti, N.; Williamson, E.; Linnane, B.; Skoric, B.; Robertson, C.F.; Robinson, P.; Massie, J.; Hall, G.L.; Sly, P.; Stick, S.; et al. Infection, inflammation, and lung function decline in infants with cystic fibrosis. Am. J. Respir. Crit. Care Med. 2011, 184, 75-81. [CrossRef]

30. Gangell, C.; Gard, S.; Douglas, T.; Park, J.; de Klerk, N.; Keil, T.; Brennan, S.; Ranganathan, S.; Robins-Browne, R.; Sly, P.D.; et al. Inflammatory responses to individual microorganisms in the lungs of children with cystic fibrosis. Clin. Infect. Dis. Off. Publ. Infect. Dis. Soc. Am. 2011, 53, 425-432. [CrossRef]

31. Amin, R.; Dupuis, A.; Aaron, S.D.; Ratjen, F. The effect of chronic infection with Aspergillus fumigatus on lung function and hospitalization in patients with cystic fibrosis. Chest 2010, 137, 171-176. [CrossRef] [PubMed]

32. Armstrong, D.A.; Nymon, A.B.; Ringelberg, C.S.; Lesseur, C.; Hazlett, H.F.; Howard, L.; Marsit, C.J.; Ashare, A. Pulmonary microRNA profiling: Implications in upper lobe predominant lung disease. Clin. Epigenetics 2017, 9, 56. [CrossRef] [PubMed]

33. Adamali, H.; Armstrong, M.E.; McLaughlin, A.M.; Cooke, G.; McKone, E.; Costello, C.M.; Gallagher, C.G.; Leng, L.; Baugh, J.A.; Fingerle-Rowson, G.; et al. Macrophage migration inhibitory factor enzymatic activity, lung inflammation, and cystic fibrosis. Am. J. Respir. Crit. Care Med. 2012, 186, 162-169. [CrossRef]

34. Rayner, R.J.; Hiller, E.J.; Ispahani, P.; Baker, M. Haemophilus infection in cystic fibrosis. Arch. Dis. Child. 1990, 65, 255-258. [CrossRef] [PubMed]

35. Wang, Z.; Ruan, Z.; Mao, Y.; Dong, W.; Zhang, Y.; Yin, N.; Jiang, L. miR-27a is up regulated and promotes inflammatory response in sepsis. Cell. Immunol. 2014, 290, 190-195. [CrossRef] [PubMed]

36. Liang, S.; Song, Z.; Wu, Y.; Gao, Y.; Gao, M.; Liu, F.; Wang, F.; Zhang, Y. MicroRNA-27b Modulates Inflammatory Response and Apoptosis during Mycobacterium tuberculosis Infection. J. Immunol. 2018, 200, 3506-3518. [CrossRef] [PubMed]

37. Ideozu, J.E.; Zhang, X.; Rangaraj, V.; McColley, S.; Levy, H. Microarray profiling identifies extracellular circulating miRNAs dysregulated in cystic fibrosis. Sci. Rep. 2019, 9, 15483. [CrossRef]

38. Luo, Q.; Zhu, J.; Zhang, Q.; Xie, J.; Yi, C.; Li, T. MicroRNA-486-5p Promotes Acute Lung Injury via Inducing Inflammation and Apoptosis by Targeting OTUD7B. Inflammation 2020. [CrossRef]

(C) 2020 by the authors. Licensee MDPI, Basel, Switzerland. This article is an open access article distributed under the terms and conditions of the Creative Commons Attribution (CC BY) license (http://creativecommons.org/licenses/by/4.0/). 